\title{
Energy Scales in X-ray Microcalorimeters
}

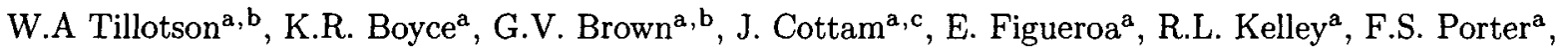
C.K. Stahle

${ }^{a} N A S A$, Goddard Space Flight Center, Greenbelt, Maryland, 20771

${ }^{\mathrm{b}}$ University of Maryland, College Park, 20742, USA

${ }^{c}$ NAS/NRC Resident Research Associate

Microcalorimeter pulse shape characteristics, such as pulse height, decay time and rise time, are dependent on the detector temperature and bias as well as the photon energy and flux. We examine the nature of the temperature dependency by illuminating the ASTRO-E2 X-ray Spectrometer (XRS) microcalorimeter array with $\mathrm{X}$-rays generated by electron impact on a range of foil targets. The resulting pulses are collected for a range of detector temperatures. We observe and model the temperature dependence of the pulse shape characteristics by fitting the data with non-linear pulse models. Our aim is to determine a robust method for correcting the energy scale obtained in ground calibration for slight differences in the operating conditions while in orbit.

2003 LTD-10 Conference Proceedings. 